\title{
Aspiração de vias aéreas artificiais após o guideline de 2010: o que mudou?
}

\author{
Artificial airway aspiration after the 2010 guideline: what has changed?
}

Aspiración de vías aéreas artificiales después de la directriz de 2010: ¿qué ha cambiado?

Aldenora Laísa Paiva de Carvalho Cordeiro ${ }^{1 *}$, Juliana da Silva Garcia Nascimento², Jordana Luiza Gouvêa de Oliveira ${ }^{2}$, Mateus Goulart Alves ${ }^{3}$, Fernanda Titareli Merizio Martins Braga ${ }^{2}$, Maria Célia Barcellos Dalri².

\section{RESUMO}

Objetivo: Sintetizar as evidências científicas para a prática da aspiração em sistema aberto, de vias aéreas artificiais de pacientes adultos e idosos, publicadas após a disponibilização do guideline de 2010. Métodos: Revisão integrativa realizada em junho de 2021 nas bases US National Library of Medicine National Institutes Database Search of Health, Cumulative Index to Nursing and Allied Health Literature, Literatura LatinoAmericana e do Caribe em Ciências da Saúde, Scopus, Embase e ERIC. Adotou-se o programa Rayyan para seleção dos estudos e a Análise Temática. Resultados: Identificaram-se 1519 estudos primários e seis compuseram a amostra. Elaboraram-se duas categorias: novos indicadores para a aspiração de vias aéreas artificiais e avanços na prática clínica da aspiração. Considerações finais: Os indicadores para realizar a aspiração descritos no guideline de 2010 foram corroborados: presença de crepitações na traqueia, curva ventilatória em formato de dente de serra, tosse ineficaz, roncos e estertores, queda de saturação, alteração do padrão respiratório, sudorese e cianose; alarmes de alta pressão e alterações nos gases arteriais. Recomendaram-se dois novos critérios para estabelecer a prática clínica da aspiração, a pré-oxigenação do paciente com oxigênio a 0,20\% acima do basal e a utilização de uma pressão de sucção de $80-120 \mathrm{mmHg}$.

Palavras-chave: Pacientes, Sucção, Intubação intratraqueal, Respiração artificial, Guias de prática clínica como assunto.

\begin{abstract}
Objective: To synthesize the existing scientific evidence on the new recommendations for the practice of aspiration of artificial airways in adult and elderly patients evidenced after the publication of the 2010 guideline. Methods: Integrative review carried out in June 2021 in the sources US National Library of Medicine National Institutes Database Search of Health, Cumulative Index to Nursing and Allied Health Literature, Latin American and Caribbean Literature in Health Sciences, Scopus, Embase and ERIC. The Rayyan program for selection and the Thematic Analysis were adopted. Results: 1519 studies were identified and six comprised the sample. Two categories were developed: new indicators for artificial airway aspiration and advances in the clinical practice of artificial airway aspiration. Final considerations: The indicators for aspiration were corroborated, consisting of the presence of crackles in the trachea, sawtooth-shaped ventilatory curve, ineffective cough, snoring and rales, drop in saturation, change in breathing pattern, sweating and cyanosis; high pressure alarms and changes in arterial gases. Two criteria were recommended for maintaining patient safety during aspiration, pre-oxygenation of the patient with oxygen at $0.20 \%$ above baseline and the use of a suction pressure of 80 $120 \mathrm{mmHg}$.
\end{abstract}

Key words: Patients, Suction, Intratracheal intubation, Artificial respiration, Clinical practice guides as topic.

\section{RESUMEN}

Objetivo: Sintetizar la evidencia científica sobre las recomendaciones para la aspiración de vía aérea artificial en pacientes adultos y ancianos evidenciadas luego de la publicación de la guía 2010. Métodos: Revisión

\footnotetext{
${ }^{1}$ Hospital de Clínicas da Universidade Federal do Triangulo Mineiro (HC-UFTM), Uberaba - MG.

*E-mail: alaisapc@hotmail.com

2Escola de Enfermagem de Ribeirão Preto da Universidade de São Paulo (EERP-USP), Ribeirão Preto - SP.

3Universidade do Estado de Minas Gerais (UEMG), Belo Horizonte - MG.
}

SUBMETIDO EM: 10/2021 
integrativa realizada en junio de 2021 en las fuentes US National Library of Búsqueda de salud en la base de datos de los Institutos Nacionales de Medicina, Índice acumulativo de literatura sobre enfermería y salud afín, Literatura Latinoamericana y del Caribe en Ciencias de la Salud, Scopus, Embase y ERIC. Se adoptaron el programa Rayyan. Resultados: Se identificaron 1519 estudios y seis conformaron la muestra. Se desarrollaron dos categorías: nuevos indicadores y avances en la práctica clínica. Consideraciones finales: Se corroboraron los indicadores de aspiración, consistente en presencia de crepitantes en la tráquea, curva ventilatoria en forma de diente de sierra, tos ineficaz, ronquidos y estertores, caída de la saturación, cambio en el patrón respiratorio, sudoración y cianosis; alarmas de alta presión y cambios en los gases arteriales. Se recomendaron dos criterios para mantener la seguridad del paciente durante la aspiración, la preoxigenación del paciente con oxígeno al 0,20\% por encima del valor inicial y el uso de una presión de succión de 80 $120 \mathrm{mmHg}$.

Palabras clave: Pacientes, Succión, Intubación intratraqueal, Respiración artificial, Guías de práctica clínica como tema.

\section{INTRODUÇÃO}

Pacientes criticamente enfermos, tratados com ventilação mecânica, requerem uma via aérea artificial, caracterizada geralmente por um tubo endotraqueal. A presença da via área artificial favorece a retenção de secreções traqueobrônquicas devido ao reflexo de tosse prejudicado, à diminuição da depuração mucociliar e ao aumento da produção de muco, fatores que exigem a prática da aspiração endotraqueal (SOLE ML, et al., 2015).

A aspiração de vias aéreas artificiais, caracterizada pela remoção de secreções do trato respiratório inferior, por meio da inserção de um cateter de aspiração e um sistema de pressão negativa, capaz de manter a permeabilidade das vias aéreas, é uma intervenção essencial para a manutenção da vida, redução do tempo de internação hospitalar e da morbimortalidade dos pacientes (BULECHEK GM, et al., 2016; CREDLAND N, 2016).

Diante da necessidade de obter-se um referencial teórico capaz de padronizar as melhores práticas para a aspiração de vias aéreas artificiais, em 2010, a American Association for Respiratory Care estabeleceu uma diretriz adotada por muitas instituições de saúde, para fundamentar os protocolos de aspiração endotraqueal e os treinamentos nesse âmbito (PINTO DM, et al., 2015; AARC, 2010).

Cabe ressaltar, no entanto que, passados 10 anos da publicação do referido guideline, não foram desenvolvidos estudos que contemplassem o impacto desta diretriz para a prática clínica e/ou sintetizassem as evidências científicas produzidas neste período, capazes de provocar alterações nas recomendações vigentes (AARC, 2010; PINTO DM, et al., 2015; JESIEN S, et al., 2019).

Esta lacuna científica instigou o questionamento: quais as novas evidências sobre aspiração de vias aéreas artificiais de pacientes adultos e idosos, ventilados mecanicamente, que surgiram após a publicação do guideline de 2010, produzido pela American Association for Respiratory Care, que podem alicerçar a prática clínica e contribuir para a segurança desta clientela?.

Este estudo objetivou sintetizar as evidências científicas para a prática da aspiração em sistema aberto, de vias aéreas artificiais de pacientes adultos e idosos, publicadas após a disponibilização do guideline de 2010

\section{MÉTODOS}

Trata-se de uma revisão integrativa de literatura que cumpriu as seguintes etapas: (1) identificação do tema e questão de norteadora; (2) busca e seleção dos estudos na literatura; (3) categorização dos estudos; (4) análise dos estudos selecionados e apresentação da revisão (WHITTEMORE R e KNAFL K, 2017).

Adotou-se a recomendação PRISMA, configurada por um checklist de 27 itens e um fluxograma de quatro etapas, que subsidiaram o rigor metodológico desta revisão (MOHER D, et al., 2009). 
Estabeleceu-se como tema a aspiração em sistema aberto, de vias aéreas artificiais, em pacientes adultos e idosos, submetidos à ventilação mecânica, intencionando compreender o que mudou na prática clínica, após a publicação do guideline de 2010 e quais recomendações foram reformuladas baseando-se na produção científica estabelecida ao longo do tempo.

A questão norteadora de pesquisa foi estruturada por meio da estratégia Patient Intervention- Outcomes (PIO), uma variação da estratégia Patient- Intervention- Comparison- Outcomes (PICO), considerando-se como acrônimo P: pacientes adultos e idosos, intubados e ventilados mecanicamente, o I: a aspiração endotraqueal por sistema aberto e como acrônimo O: os avanços na prática clínica da aspiração de vias aéreas artificiais após o guideline de 2010 (MENDES KD, et al., 2008).

Elaborou-se a pergunta: Quais são as novas recomendações sugeridas pela literatura científica quanto a prática clínica da aspiração de vias aéreas artificiais em sistema aberto, de pacientes adultos e idosos, ventilados mecanicamente, após a publicação do guideline de 2010?

Os critérios de inclusão foram: artigos científicos provenientes de estudos primários; publicados nos últimos 11 anos; recorte temporal justificado pelo ano de publicação do último guideline para aspiração de vias aéreas artificiais (2010), sem delimitação de idioma; que apresentassem alterações ou novas recomendações quanto aos critérios que embasam a prática clínica da aspiração de vias aéreas artificiais, por sistema aberto, em adultos e/ou idosos, intubados e em ventilação mecânica. Foram excluídas revisões da literatura, estudos de caso, dissertações, teses, monografias e resumos publicados em anais de eventos.

Realizou-se a busca dos estudos em junho de 2021 nas fontes de informação: US National Library of Medicine National Institutes Database Search of Health (PubMed $\left.{ }^{\circledR}\right)$, SCOPUS, Cumulative Index to Nursing and Allied Health Literature (CINAHL), Literatura Latino-Americana e do Caribe em Ciências da Saúde (LILACS); EMBASE e Educational Resources Information Center (ERIC).

$\mathrm{Na}$ PubMed ${ }^{\circledR}$ selecionou-se os seguintes descritores controlados e seus sinônimos, na língua inglesa, identificados no Medical Subjects Headings (MeSH): Patients; Suction; Intubation; Intratracheal; Respiration, Artificial, e realizou-se a seguinte estratégia: (("patients"[MeSH Terms] OR "patients"[All Fields]) OR ("patients"[MeSH Terms] OR "patients"[All Fields] OR "patient"[All Fields]) OR ("patients"[MeSH Terms] OR "patients"[All Fields] OR "clients"[All Fields]) OR ("patients"[MeSH Terms] OR "patients"[All Fields] OR "client"[All Fields]) AND ("suction"[MeSH Terms] OR "suction"[All Fields]) OR ("suction"[MeSH Terms] OR "suction"[All Fields] OR "suctions"[All Fields]) OR "Aspiration, Mechanical"[All Fields] OR "Aspirations, Mechanical"[All Fields] OR "Mechanical Aspiration"[All Fields] OR "Mechanical Aspirations"[All Fields] OR "Drainage, Suction"[All Fields] OR "Drainages, Suction"[All Fields] OR "Suction Drainage"[All Fields] OR "Suction Drainages"[All Fields] AND "Intubation, Intratracheal"[All Fields] OR "Intratracheal Intubation"[All Fields] OR "Intratracheal Intubations"[All Fields] OR "Intubation, Endotracheal"[All Fields] OR "Endotracheal Intubation"[All Fields] OR "Endotracheal Intubations"[All Fields] AND "Respiration, Artificial"[All Fields] OR "Artificial Respiration"[All Fields] OR "Artificial Respirations"[All Fields] OR "Respirations, Artificial"[All Fields] OR "Ventilation, Mechanical"[All Fields] OR "Mechanical Ventilations"[All Fields] OR "Ventilations, Mechanical"[All Fields] OR "Mechanical Ventilation")).

$\mathrm{Na}$ Scopus determinaram-se os descritores controlados na língua inglesa: Patients; Suction; Intubation; Intratracheal; Respiration, Artificial, e a estratégia de busca: TITLE-ABS-KEY( patients OR patient OR clients OR client) AND ( suction OR suctions OR "Aspiration, Mechanical" OR "Aspirations, Mechanical" OR "Mechanical Aspiration" OR "Mechanical Aspirations" OR "Drainage, Suction" OR "Drainages, Suction" OR "Suction Drainage" OR "Suction Drainages") AND ("Intubation, Intratracheal" OR "Intratracheal Intubation" OR "Intratracheal Intubations" OR "Intubations, Intratracheal" OR "Intubation, Endotracheal" OR "Endotracheal Intubation" OR "Endotracheal Intubations" OR "Intubations, Endotracheal") AND ("Respiration, Artificial" OR "Artificial Respiration" OR "Artificial Respirations" OR "Respirations, Artificial" OR "Ventilation, Mechanical" OR "Mechanical Ventilations" OR "Ventilations, Mechanical" OR "Mechanical Ventilation"). 
$\mathrm{Na}$ CINAHL os descritores controlados foram identificados em Títulos, na língua inglesa e nas suas versões em português e espanhol: Patients; Suction; Intubation, Intratracheal; Respiration, Artificial. Procedeu-se a estratégia: SU ((Patients AND Suction (OR "Suctioning, Endotracheal") AND "Respiration, Artificial"))

Na LILACS os descritores controlados foram selecionados nos Descritores de Ciências da Saúde (Decs), em português: Pacientes; Sucção; "Intubação Intratraqueal"; "Respiração Artificial", e em suas versões em inglês e espanhol. Realizou-se a estratégia: MH ((Patients AND Suction AND "Intubation, Intratracheal" AND "Respiration, Artificial")) Espanhol: MH ((Pacientes AND Succión AND "Intubación Intratraqueal" AND "Respiración Artificial")) Português: MH ((Pacientes AND Sucção AND "Intubação Intratraqueal" AND "Respiração Artificial")).

$\mathrm{Na}$ EMBASE os descritores, controlados na língua inglesa foram: Patients; Suction; Intubation, Intratracheal; Respiration, Artificial. Procedeu-se a estratégia: (patients OR patient OR clients OR client) AND (suction OR suctions OR 'aspiration, mechanical' OR 'aspirations, mechanical' OR 'mechanical aspiration' OR 'mechanical aspirations' OR 'drainage, suction' OR 'drainages, suction' OR 'suction drainage' OR 'suction drainages') AND ('intubation, intratracheal' OR 'intratracheal intubation' OR 'intratracheal intubations' OR 'intubations, intratracheal' $O R$ 'intubation, endotracheal' $O R$ 'endotracheal intubation' $O R$ 'endotracheal intubations' OR 'intubations, endotracheal') $A N D$ ('respiration, artificial' OR 'artificial respiration' OR 'artificial respirations' $O R$ 'respirations, artificial' $O R$ 'ventilation, mechanical' OR 'mechanical ventilations' $O R$ 'ventilations, mechanical' OR 'mechanical ventilation').

Na ERIC os descritores, controlados na língua inglesa foram: Patients; Suction; Intubation, Intratracheal; Respiration, Artificial. Determinou-se a seguinte estratégia de busca: (patients $O R$ patient $O R$ clients $O R$ client) AND (suction OR suctions OR 'aspiration, mechanical' OR 'aspirations, mechanical' OR 'mechanical aspiration' OR 'mechanical aspirations' OR 'drainage, suction' OR 'drainages, suction' OR 'suction drainage' $O R$ 'suction drainages') $A N D$ ('intubation, intratracheal' $O R$ 'intratracheal intubation' OR 'intratracheal intubations' OR 'intubations, intratracheal' OR 'intubation, endotracheal' OR 'endotracheal intubation' OR 'endotracheal intubations' OR 'intubations, endotracheal') AND ('respiration, artificial' OR 'artificial respiration' $O R$ 'artificial respirations' OR 'respirations, artificial' OR 'ventilation, mechanical' OR 'mechanical ventilations' OR 'ventilations, mechanical' OR 'mechanical ventilation').

Dos 1519 estudos identificados, a priori, removeu-se 648 artigos duplicados. Após, procedeu-se a aplicação dos critérios de elegibilidade, a partir da leitura dos títulos e resumos, por dois pesquisadores, de forma independente, por meio de um programa de revisão gratuito da web de versão única chamado Rayyan Qatar Computing Research Institute (Rayyan QCRI). Este programa proporciona que os autores de revisões de literatura executem a seleção de estudos de forma simples, organizada e rápida, e permite exportar os artigos de uma base de dados determinada para o programa, excluir as referências duplicatas, com o cegamento do pesquisador auxiliar, o que garante a confiabilidade e precisão metodológica deste processo (OUZZANI M, et al., 2016).

O total de 37 pesquisas foi entregue a um terceiro pesquisador, visto as divergências de avaliação, para inclusão ou exclusão. Após, procedeu-se à leitura na íntegra de 125 artigos, para definir a amostra final. Para extração dos dados foi empregado um instrumento validado e adaptado, que contemplou os critérios: autores; ano de publicação; origem do estudo; objetivo e detalhamento metodológico; principais resultados/conclusão (URSI ES e GALVÃO CM, 2006).

Os achados foram analisados de acordo com os pressupostos da Análise Temática e foram percorridas três etapas, a saber: pré-análise, caracterizada pela leitura e organização das informações convergentes ou unidades de registro; exploração do material que consistiu no agrupamento minucioso das unidades de registro; e tratamento dos dados, estabelecendo-se as categorias (MINAYO MC, 2017). Posteriormente, o nível de evidência foi classificado (MELNYK BM e FINEOUT-OVERHOLT E, 2011). O fluxograma de seleção dos estudos foi elaborado seguindo as recomendações Preferred Reporting Items for Systematic Reviews and Meta-Analyses (PRISMA) (MOHER D, et al., 2009). Em consonância com os aspectos éticos e legais da Resolução no 466/2012, a pesquisa não foi submetida ao Comitê de Ética em Pesquisa, por se tratar de uma revisão da literatura e não envolver seres humanos. 


\section{RESULTADOS}

Consideraram-se elegíveis para compor a amostra final da presente pesquisa, seis estudos primários. 0 processo de seleção está apresentado na Figura 1.

Figura 1 - Fluxograma de seleção dos estudos para compor a amostra da revisão integrativa segundo PRISMA.

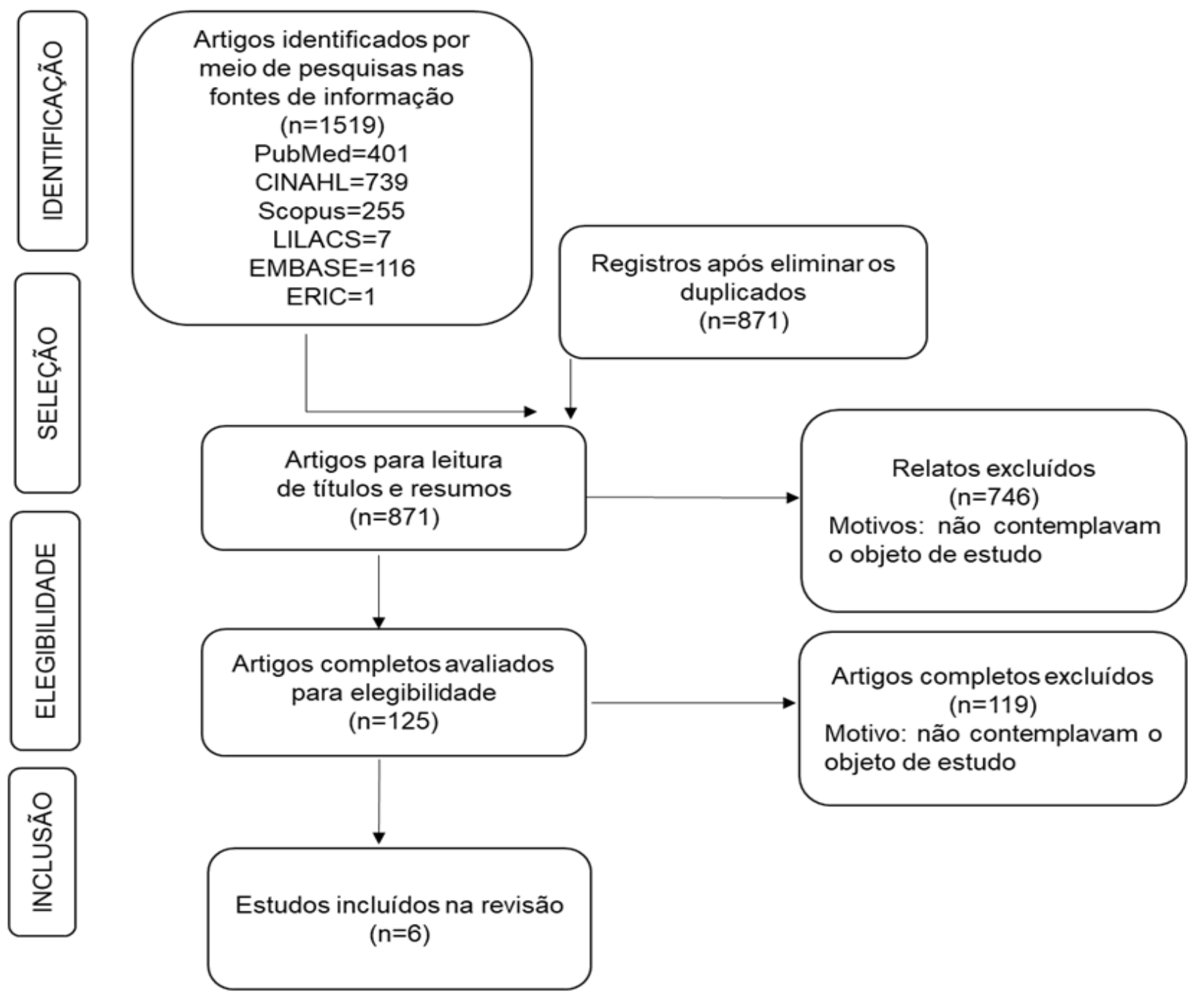

Fonte: Cordeiro ALPC, et al., 2021.

Os estudos pertencentes a amostra de artigos selecionada, foram caracterizados, descrevendo-se os autores, ano de publicação, objetivo, método, principais resultados, conclusão e nível de evidência, como se observa no Quadro 1.

Os estudos que compuseram a amostra da presente pesquisa, foram publicados, na maioria, em $2015 \mathrm{e}$ 2019 (SOLE ML, et al., 2015; BELL L, 2015; JESIEN S, et al., 2019; GILDER E, et al., 2019). A metade nacional e do tipo descritivo, com nível de evidência 6 (SOLE ML, et al., 2015; GILDER E, et al., 2019; BELL L, 2015; BALBINO CM, et al., 2016; JESIEN S, et al., 2019; VIANNA JR, et al., 2017).

Diante da análise dos achados elaboraram-se duas categorias: (1) novos indicadores para a aspiração de vias aéreas artificiais, que contemplou os critérios clínicos necessários para proceder a aspiração endotraqueal e (2) avanços na prática clínica da aspiração de vias aéreas artificiais, abordando as alterações nas recomendações vigentes, após o guideline de 2010. 
Quadro 1 - Apresentação da caracterização dos estudos incluídos na revisão integrativa de literatura.

\begin{tabular}{|c|c|c|}
\hline Autores/ ano & Objetivo e detalhamento metodológico & Principais resultados, conclusão e Nível de Evidência \\
\hline $\begin{array}{l}\text { SOLE ML, et al., } \\
2015\end{array}$ & $\begin{array}{l}\text { Determinar os critérios para a aspiração endotraqueal } \\
\text { em pacientes ventilados mecanicamente. Estudo } \\
\text { descritivo, realizado com } 42 \text { pacientes. Após a aspiração } \\
\text { endotraqueal, os pacientes foram avaliados de hora em } \\
\text { hora, até } 4 \text { horas, identificando-se critérios indicativos de } \\
\text { aspiração. }\end{array}$ & $\begin{array}{l}\text { Os critérios para aspiração mais frequentes foram: crepitações na traqueia } \\
(88 \%) \text {, forma de onda de dente de serra no ventilador mecânico (33\%), tosse } \\
(29 \%) \text { e secreções visíveis }(5 \%) \text {. Foram corroborados os critérios indicativos } \\
\text { de necessidade da aspiração, apontados pelo guideline de } 2010 \text {, como } \\
\text { presença de secreção endotraqueal, crepitações auscultadas sobre a região } \\
\text { da traqueia; presença de um padrão de onda serrilhada no ventilador mecânico; } \\
\text { alarmes de alta pressão e tosse. Nível de Evidência: } 6\end{array}$ \\
\hline BELL L, 2015 & $\begin{array}{l}\text { Descrever as melhores práticas quanto à aspiração } \\
\text { endotraqueal. Estudo descritivo, que aborda as melhores } \\
\text { práticas quanto a aspiração endotraqueal de pacientes } \\
\text { intubados e ventilados mecanicamente. }\end{array}$ & $\begin{array}{l}\text { Descreve-se a ausculta de crepitações grossas sobre a traqueia como } \\
\text { indicador mais comum para suçãa, e recomenda-se as seguintes práticas } \\
\text { avaliar a equipe de enfermagem e de fisioterapia quanto as indicações para a } \\
\text { aspiração; estabelecer uma prática baseada em evidencias para as unidades } \\
\text { de saúde sobre aspiração; analisar e validar os protocolos existentes de } \\
\text { aspiração e incluir diretrizes estabelecidas, estabelecer um processo de revisão } \\
\text { por pares para competências relacionado à aspiração, divulgar as atualizações } \\
\text { dos protocolos. Concluiu-se que se deve evitar a prática de aspiração realizada } \\
\text { em intervalos de rotina, a instilação de soro fisiológico na cânula endotraqueal. } \\
\text { Nível de Evidência: } 6\end{array}$ \\
\hline $\begin{array}{l}\text { BALBINO CM, et al., } \\
2016\end{array}$ & $\begin{array}{l}\text { Identificar o conhecimento dos profissionais de } \\
\text { enfermagem sobre a aspiração. Estudo descritivo, } \\
\text { realizado com } 15 \text { profissionais de enfermagem. Para a } \\
\text { coleta de dados, optou-se pela técnica de observação } \\
\text { estruturada não participante e um diário de campo. }\end{array}$ & $\begin{array}{l}\text { Foram observados } 50 \text { procedimentos de aspiração, sendo } 46 \%(n=23) \text { no } \\
\text { período diurno e } 54 \% \text { ( } n=27 \text { ) no noturno. Concluiu-se que, a elaboração de uma } \\
\text { estratégia de educação continuada e sua aderência pelos profissionais de } \\
\text { enfermagem, pode nortear a sua atuação de acordo com os protocolos que } \\
\text { regem as práticas de aspiração endotraqueal. Ainda há lacunas de } \\
\text { conhecimento quanto ao procedimento, principalmente em relação ao diâmetro } \\
\text { ideal da sonda de aspiração, pressão de sucção e outros. } \\
\text { Nível de Evidência: } 6\end{array}$ \\
\hline
\end{tabular}




\begin{tabular}{|c|c|c|}
\hline Autores/ ano & Objetivo e detalhamento metodológico & Principais resultados, conclusão e Nível de Evidência \\
\hline $\begin{array}{l}\text { VIANNA JR, et al., } \\
2017\end{array}$ & $\begin{array}{l}\text { Comparar a eficácia da hiperoxigenação com } \mathrm{FIO}_{2} 0,20 \\
\text { acima da linha de base e hiperoxigenação com } \mathrm{FIO}_{2} 1,0 \text {, } \\
\text { na prevenção da hipoxemia durante a aspiração } \\
\text { endotraqueal. Estudo clínico randomizado, conduzido } \\
\text { com } 68 \text { indivíduos, ventilados mecanicamente com } \mathrm{FIO}_{2} \\
<0,60 \text {. Aspiração endotraqueal aberta foi realizada } \\
\text { usando } 2 \text { sequências de intervenção diferentes: } \\
\text { hiperoxigenação de } 0,20 \text { acima da linha de base } \mathrm{FIO}_{2} \\
\left(\mathrm{FIO}_{2} 0,20\right) \text { e hiperoxigenação } 1,0\left(\mathrm{FIO}_{2} 1,0\right) \text {. }\end{array}$ & $\begin{array}{l}\text { Concluiu-se que, em indivíduos adultos ventilados mecanicamente, a } \\
\text { hiperoxigenação com } \mathrm{FIO}_{2} \text { 0,20 acima da linha de base evita a hipoxemia. } \\
\text { Nível de Evidência: } 2\end{array}$ \\
\hline $\begin{array}{l}\text { GILDER E, et al., } \\
2019\end{array}$ & $\begin{array}{l}\text { Descrever a prática de aspiração endotraqueal de } \\
\text { enfermeiras em uma Unidade de Terapia Intensiva na } \\
\text { Nova Zelândia e Austrália, incluindo os critérios para } \\
\text { realizar o procedimento. Estudo observacional. Todos os } \\
\text { pacientes adultos, admitidos até às 10:00 no dia do } \\
\text { estudo, foram incluídos. Avaliou-se a indicação para } \\
\text { aspiração, a pressão de sucção, uso de pré-oxigenação, } \\
\text { medidas de oxigenação e aspiração na extubação. }\end{array}$ & $\begin{array}{l}\text { Um total de } 1891 \text { eventos foi observado em } 227 \text { pacientes durante o dia do } \\
\text { estudo, uma média de oito intervenções por paciente. Os principais critérios } \\
\text { para aspirar foram: secreções audíveis (63\%) e visíveis (39\%). A pressão } \\
\text { média do recipiente de sucção foi de } 337 \mathrm{mmHg} .67 \% \text { dos pacientes receberam } \\
\text { pré-oxigenação e a aspiração na extubação foi realizada por } 84 \% \text { dos } \\
\text { enfermeiros. Concluiu-se que, algumas práticas foram inconsistentes com as } \\
\text { diretrizes internacionais, em particular no que diz respeito avaliação do } \\
\text { paciente para aspiração e critérios para realizar a aspiração. } \\
\text { Nível de Evidência: } 6\end{array}$ \\
\hline $\begin{array}{c}\text { JESIEN S, et al., } \\
2019\end{array}$ & $\begin{array}{l}\text { Avaliar a técnica de aspiração de vias aéreas em } \\
\text { pacientes ventilados mecanicamente, em duas Unidades } \\
\text { de Terapia Intensiva e analisar o impacto de uma } \\
\text { intervenção educacional voltada a profissionais de } \\
\text { saúde. Estudo do tipo coorte, com } 36 \text { profissionais. O } \\
\text { procedimento foi observado por dois avaliadores e a } \\
\text { intervenção realizada da mesma forma nas duas } \\
\text { unidades. Como foi a intervenção educacional? Em que } \\
\text { momento foi realizado? } \\
\text { As instituições participantes são uma pública e privada? }\end{array}$ & $\begin{array}{l}\text { Quando questionados quanto à indicação da aspiração, a resposta } \\
\text { predominante nas duas instituições }(55,5 \% \text { dos casos) foi a rotina. Houve uma } \\
\text { piora considerável na utilização no hospital privado quanto ao uso de EPI } \\
(0,006) \text {, proteção do látex da extensão para aspiração }(0,03) \text { e uso da água } \\
\text { destilada para lavagem desta extensão }(0,0001) \text {. No hospital público notou-se } \\
\text { uma piora significativa quanto ao descarte do soro fisiológico após o } \\
\text { procedimento }(0,0001) \text {. Concluiu-se que, a intervenção educacional foi } \\
\text { significativamente eficaz no hospital público, apoiando que a aspiração não } \\
\text { deve ser padronizada e realizada como um procedimento rotineiro, mas sim } \\
\text { sob a avaliação de critérios específicos. Nível de Evidência: } 4\end{array}$ \\
\hline
\end{tabular}

Fonte: Cordeiro ALPC, et al., 2021. 
O Quadro 2, apresenta as diretrizes do guideline 2010 para aspiração de vias aéreas artificiais de pacientes adultos, intubados e ventilados mecanicamente que foram investigadas ao longo do tempo, pelos estudos científicos, obtendo-se novas recomendações para a prática clínica da aspiração.

Quadro 2 - Diretrizes para aspiração de vias aéreas artificiais investigadas pelos estudos científicos após a publicação do guideline 2010, para aspiração de vias aéreas artificiais de pacientes adultos, e as novas recomendações.

\begin{tabular}{|c|c|c|}
\hline $\begin{array}{l}\text { Principais recomendações do } \\
\text { guideline } 2010 \text { para aspiração de } \\
\text { vias aéreas artificiais de pacientes } \\
\text { adultos, intubados e ventilados } \\
\text { mecanicamente. }\end{array}$ & $\begin{array}{c}\text { Categoria 1: Novos } \\
\text { indicadores para a aspiração } \\
\text { de vias aéreas artificiais }\end{array}$ & $\begin{array}{l}\text { Categoria 2: Avanços na } \\
\text { prática clínica da } \\
\text { aspiração de vias aéreas }\end{array}$ \\
\hline $\begin{array}{l}\text { 1-Recomenda-se realizar a aspiração } \\
\text { endotraqueal quando houver presença } \\
\text { de: padrão serrilhado na curva de } \\
\text { fluxo-volume na tela do monitor do } \\
\text { ventilador e / ou presença de estertores } \\
\text { grossos sobre a traqueia; aumento da } \\
\text { pressão inspiratória de pico no modo } \\
\text { ventilatório controlado por volume ou } \\
\text { diminuição do volume corrente, no } \\
\text { modo ventilatório controlado por } \\
\text { pressão; diminuição da saturação de } \\
\text { oxigênio e / ou alteração nos valores de } \\
\text { gases arteriais; secreções visíveis nas } \\
\text { vias aéreas; tosse ineficaz; dificuldade } \\
\text { respiratória aguda; suspeita de } \\
\text { aspiração de secreções das vias } \\
\text { aéreas superiores ou secreções } \\
\text { gástricas. }\end{array}$ & $\begin{array}{l}\text { 1- Recomendou-se realizar a } \\
\text { aspiração endotraqueal na } \\
\text { presença de: crepitações na } \\
\text { traqueia; curva ventilatória em } \\
\text { formato de dente de serra; tosse } \\
\text { ineficaz; roncos e estertores } \\
\text { grossos; queda de saturação; } \\
\text { alteração do padrão respiratório, } \\
\text { sudorese e cianose; alarmes de } \\
\text { alta pressão no ventilador } \\
\text { mecânico e alterações nos } \\
\text { gases arteriais (SOLE ML, et al., } \\
\text { 2015; BELL L, 2015; VIANNA } \\
\text { JR, et al., 2017; GILDER E, et al., } \\
\text { 2019; JESIEN S, et al., 2019). }\end{array}$ & - \\
\hline $\begin{array}{l}\text { 2- Considerar a pré-oxigenação do } \\
\text { paciente com uma fração inspirada de } \\
\text { oxigênio a } 100 \% \text {, especialmente } \\
\text { quando este apresentar hipoxemia } \\
\text { antes da aspiração ou uma redução } \\
\text { clinicamente importante da saturação } \\
\text { de oxigênio com a aspiração. }\end{array}$ & - & $\begin{array}{l}\text { 2-Recomendou-se, apartir de } \\
2017 \text {, pré-oxigenar o } \\
\text { paciente usando oxigênio a } \\
0,2 \text { acima do basal já } \\
\text { programado (VIANNA JR, et } \\
\text { al., 2017). }\end{array}$ \\
\hline $\begin{array}{l}\text { 3- A pressão de sucção não deve } \\
\text { exceder } 150 \mathrm{mmHg} \text { negativos }\end{array}$ & - & $\begin{array}{l}\text { 3- Recomendou-se a partir de } \\
\text { 2016, empregar uma pressão } \\
\text { de sucção de } 80-120 \mathrm{mmHg} \\
\text { para aspirar o paciente e não } \\
\text { exceder 150mmHg (GILDER } \\
\text { E, et al., 2019; BALBINO CM, } \\
\text { et al., 2016). }\end{array}$ \\
\hline
\end{tabular}

Fonte: Cordeiro ALPC, et al., 2021. Baseado no Guideline da American Association for Respiratory Care (2010).

Das 10 principais diretrizes que compõem o Guideline da American Association for Respiratory Care de 2010, três foram investigadas ao longo do tempo, obtendo-se novas sugestões para a prática clínica da aspiração, caracterizadas por: (1) inclusão de critérios que indicam e ampliam a necessidade de aspiração 
endotraqueal, embasados, na maioria por estudos de nível de evidência 6 (SOLE ML, et al., 2015; GILDER E, et al., 2019; BELL L, 2015); (2) recomendações quanto a pré-oxigenação do paciente antes da aspiração, nível de evidência 2 (VIANNA JR, et al., 2017); (3) recomendações quanto a pressão de sucção adotada para aspiração, nível de evidência 6 (GILDER E, et al., 2019; BALBINO CM, et al., 2016).

\section{DISCUSSÃO}

Apesar da vasta produção sobre a temática da aspiração endotraqueal, desenvolvida principalmente, após a publicação do guideline de 2010 , os estudos que propuseram explorar as recomendações deste referencial e apresentaram achados que sugerem novas práticas voltadas para a aspiração endotraqueal, são ainda, incipientes e sustentados, na maioria, por manuscritos de baixo nível de evidência, condição que instiga a elaboração de estudos, capazes de avaliar a efetividade das recomendações sobre a aspiração para a segurança dos pacientes (GILDER E, et al., 2019; BELL L, 2015; BALBINO CM, et al., 2016).

Desta forma, este estudo confere ineditismo à ciência em saúde e enfermagem por apresentar a indicação de novos critérios para a aspiração de vias aéreas artificiais e os possíveis avanços na prática clínica da aspiração, baseados nas recomendações sugeridas após o guideline de 2010, fomentando a reflexão/discussão de estudantes e profissionais sobre esta temática, para direcionar a elaboração de protocolos, treinamentos nas instituições de saúde e novas pesquisas científicas (SOLE ML, et al., 2015; BELL L, 2015)

Quanto aos critérios que indicam a necessidade da aspiração de vias aéreas artificiais, foi possível ratificar os critérios estabelecidos pelo guideline de 2010. Um estudo descritivo realizado com pacientes adultos em ventilação mecânica, em unidades de terapia intensiva, identificou que, a necessidade de aspiração endotraqueal não se configura somente pela presença de secreção visível, mas também, por crepitações audíveis na região da traqueia, padrão de onda serrilhada, alarmes de alta pressão no ventilador mecânico e incapacidade do paciente de gerar uma tosse espontânea eficaz. (SOLE ML, et al., 2015).

Uma pesquisa descritiva, realizada em 2015, que apontou as melhores práticas quanto à aspiração endotraqueal, concluindo principalmente que, há uma variedade de fatores clínicos que indicam a necessidade de aspiração endotraqueal em um paciente adulto, não somente a presença de secreção visível, exigindo o desenvolvimento de protocolos, baseados em evidencias, revisado por pares, para implementação nas instituições de saúde e treinamentos dos profissionais (BELL L, 2015).

A necessidade de avaliação minuciosa e a detecção precisa de sinais clínicos que indicam a aspiração, além de capacidade técnica para condutas emergenciais em casos necessários, são um ponto de relevância destacado pela literatura (SOLE ML, et al., 2015; YOUSEFNIA-DARZI F, et al., 2016).

Nesta perspectiva, o parecer do Conselho Federal de Enfermagem (COFEN) 0557/2017 recomenda que, pacientes submetidos à intubação endotraqueal, sejam submetidos a uma avaliação rigorosa, realizada pelo profissional enfermeiro, quanto a necessidade de aspiração para estabelecer a melhor tomada de decisão e evitar a realização deste procedimento sem critério clínico e de forma rotineira, em consonância com os achados nos estudos desta revisão (JESIEN S, et al., 2019; GILDER E, et al., 2019).

Novas recomendações foram estabelecidas para a prática da aspiração, sugerindo avanços nesta temática, como também a necessidade de maiores investigações. Os valores estabelecidos para a préoxigenação dos pacientes que necessitam de aspiração endotraqueal, foram questionados por um estudo clínico, randomizado, conduzido com 68 indivíduos no Brasil. Foi comparada a eficácia da pré-oxigenação com uma $\mathrm{FIO} 2$ de $0,20 \%$ acima da linha de base, em um grupo intervenção, e a pré-oxigenação com uma $\mathrm{FIO} 2$ de $1,00 \%$, em um grupo controle. Foi constatado que, em indivíduos adultos ventilados mecanicamente, a pré-oxigenação com uma $\mathrm{FIO} 2$ de $0,20 \%$ acima da linha de base é suficiente para evitar a hipoxemia durante a aspiração endotraqueal sugerindo uma nova recomendação, diferente do proposto pelo guideline de 2010 . No entanto, esta recomendação ainda é incipiente e necessita de mais investigações (VIANNA JR, et al., 2017). 
Um estudo científico realizado em unidades de terapia intensiva na Austrália, em 2019, identificou que a prática clínica da pré-oxigenação do paciente antes da aspiração endotraqueal e o valor da FIO2, não eram alinhados entre os profissionais, pois cada unidade seguia um protocolo clínico diferente e optava por níveis variados de oxigênio. Sugere-se que esta prática seja padronizada e baseada em evidências (GILDER E, et al., 2019).

A pressão de sucção ideal para aspiração endotraqueal também foi abordada pela literatura, após a publicação do guideline de 2010. Uma pressão de aspiração baixa, entre 80 a $120 \mathrm{mmHg}$ é indicada para a redução do risco de atelectasia, hipóxia e danos à mucosa traqueal do paciente, contudo, na prática clínica, este critério geralmente não é avaliado, ficando o profissional livre para alterar a pressão de aspiração da maneira que achar necessário. O guideline 2010 recomendou uma sucção menor que $150 \mathrm{mmHg}$ e esta revisão da literatura evidenciou uma recomendação ainda menor (YOUSEFNIA-DARZI F, et al., 2016; LOPES VJ, et al., 2018; PEDERSEN CM, et al., 2009).

Uma pesquisa observacional realizada em unidades de terapia intensiva na Nova Zelândia e Austrália, em 2019, avaliou um total de 1891 eventos durante a aspiração de vias aéreas artificiais de 227 pacientes gravemente enfermos e identificou que, a pressão média do recipiente de sucção foi de $337 \mathrm{mmHg}$, considerada elevada e danosa para a integridade da mucosa respiratória. Este estudo sugeriu a elaboração de protocolos assistenciais e pesquisas científicas frente a exigência de melhores práticas no âmbito da aspiração endotraqueal (GILDER E, et al., 2019).

A disponibilidade de evidências de alta qualidade sobre a aspiração de vias aéreas continua a apresentar desafios para os profissionais da saúde, além disso, as diretrizes que estão disponíveis não estão sendo implementadas na prática clínica (GILDER E, et al., 2019).

Nesta revisão de literatura, as principais limitações identificadas foram as seguintes: abrangência da aspiração de vias aéreas somente em sistema aberto e o baixo nível de evidência da maioria dos manuscritos incluídos que dificultam a sustentação de novas práticas para a aspiração endotraqueal, a partir das recomendações estabelecidas pela literatura, após a publicação do guideline de 2010. Sugere-se desta forma, o desenvolvimento de novos estudos, metodologicamente bem desenhados e com maior nível de evidencia, capazes de corroborar ou contradizer as novas recomendações, identificadas na presente pesquisa.

\section{CONSIDERAÇÕES FINAIS}

Este estudo sintetizou o conhecimento sobre as novas recomendações para aspiração endotraqueal, sugeridas pela literatura científica após a publicação do guideline 2010, corroborando os critérios que indicam a necessidade de aspiração, configurados principalmente pela presença de crepitações na traqueia, curva ventilatória em formato de dente de serra, tosse, roncos e estertores grossos, queda de saturação, alteração do padrão respiratório, sudorese e cianose; alarmes de alta pressão no ventilador mecânico e alterações nos gases arteriais. Recomendou-se a pré-oxigenação do paciente adotando-se uma fração de oxigênio a 0,20\% acima do basal já programado no ventilador mecânico, e a manutenção de uma pressão de sucção de 80 $120 \mathrm{mmHg}$ durante a aspiração endotraqueal.

\section{REFERÊNCIAS}

1. AMERICAN ASSOCIATION OF RESPIRATORY CARE (AARC). AARC clinical practice guideline: endotracheal suctioning of mechanically ventilated patients with artificial airways 2010. Respiratory Care, 2010; 55(6): 758-64.

2. BALBINO CM, et al. Avaliação da técnica de aspiração de paciente em ventilação mecânica realizada pela enfermagem. Revista de Enfermagem UFPE online, 2016; 10(6): 4797-803.

3. BELL L. Using Endotracheal Suctioning for Patients. American Journal of Critical Care, 2015; 24(4): 326.

4. BULECHEK GM, et al. Classificação das Intervenções de Enfermagem (NIC). 6ํe ed. Rio de Janeiro: Elsevier, 2016.

5. CONSELHO FEDERAL DE ENFERMAGEM (COFEN). Resolução № 0557/2017. 2017. Disponível em: www.cofen.gov.br/resolucao-cofen-no05572017_54939.html. Acessado em: 08 de setembro de 2021.

6. CREDLAND N. How to perform open tracheal suction via endotracheal tube. Nursing Standard, 2016; 30(35): 36-8. 
7. GILDER E, et al. Australian and New Zealand Intensive Care Society Clinical Trials Group and The George Institute for Global Health. Endotracheal suction in intensive care: A point prevalence study of current practice in New Zealand and Australia. Australian Critical Care, 2019; 32(2): 112-5.

8. GILDER E, et al. Australian and New Zealand Intensive Care Society Clinical Trials Group and The George Institute for Global Health. Endotracheal suction in intensive care: A point prevalence study of current practice in New Zealand and Australia. Australian Critical Care. 2019; 32(2): 112-5.

9. JESIEN S, et al. Efeitos de uma intervenção educacional na adequação da aspiração endotraqueal em unidades de terapia intensiva no rio grande do sul. Revista Científica da Saúde, 2019; 1(1): 60-71.

10. LOPES VJ, et al. Aspiração endotraqueal em pacientes com via aérea artificial sob ventilação mecânica invasiva internados em UTI. Revista de Enfermagem do Centro Oeste Mineiro. 2018; 8: e1973.

11. MELNYK BM, FINEOUT-OVERHOLT E. Evidence-based practice in nursing \& healthcare: a guide to best practice. 2. ed. Philadelphia: Wolters Kluwer Health/Pippincott Williams \& Wilkins; 2011. 137 p.

12. MENDES KD, et al. Revisão integrativa: método de pesquisa para a incorporação de evidências na saúde e na enfermagem. Texto \& contexto enfermagem, 2008; 17(4): 758-64.

13. MINAYO MC. Sampling and saturation in qualitative research: consensuses and controversies. Revista Pesquisa Qualitativa, 2017; 5(7): 1-12.

14. MOHER D, et al. Preferred reporting items for systematic reviews and metaanalyses: the PRISMA statement. PLoS Medicine, 2009; 6(7): e1000097.

15. OUZZANI M, et al. Rayyan-a web and mobile app for systematic reviews. Systematic Reviews, 2016; 5(1): 210.

16. PEDERSEN CM, et al. Endotracheal suctioning of the adult intubated patient-what is the evidence? Intensive and Critical Care Nursing. 2009; 25(1): 21-30.

17. PINTO DM, et al. Segurança do paciente e a prevenção de lesões cutaneomucosas associadas aos dispositivos invasivos nas vias aéreas. Revista da Escola de Enfermagem da USP, 2015; 49(5): 775-82.

18. SOLE ML, et al. Clinical Indicators for Endotracheal Suctioning in Adult Patients Receiving Mechanical Ventilation. American Journal of Critical Care, 2015; 24(4): 318-24.

19. URSI ES, GALVÃO CM. Prevenção de lesões de pele no perioperatório: revisão integrativa da literatura. Revista Latino-Americana de Enfermagem, 2006; 14(1): 124-31.

20. VIANNA JR, et al. Comparing the Effects of Two Different Levels of Hyperoxygenation on Gas Exchange During Open Endotracheal Suctioning: A Randomized Crossover Study. Respiratory Care, 2017; 62(1): 92-101.

21. WHITTEMORE R, KNAFL K. The integrative review: updated methodology. Journal of Advanced Nursing, 2005; 52(5): 546-53.

22. YOUSEFNIA-DARZI F, et al. Effects of thoracic squeezing on airway secretion removal in mechanically ventilated patients. Iranian Journal of Nursing and Midwifery Research, 2016; 21(3): 337-42. 